\title{
Erratum
}

Visc Med 2017;33:226

DOI: $10.1159 / 000477195$

In the article by Kirstein MM, Vogel A:

\section{Epidemiology and Risk Factors of Cholangiocarcinoma.}

Visc Med 2016;32:395-400 the legend of figure 1 was incorrectly given.

The correct legend is as follows:

Fig. 1. Global incidence rates of CCA (originally published by Banales et al. [71] (www.nature.com/nrgastro/journal/v13/n5/full/nrgastro.2016.51.html); Creative Commons Attribution 4.0 International License (http://creativecommons.org/licenses/by/4.0/)). 